\title{
Analysis of Shupe Effect of Fiber Optic Ring Resonator Based on Photonic Crystal Fiber
}

\author{
Bo Yang, Yong Li, Li Sun \\ Beijing Institute of Control Engineering, Beijing, China \\ Email: yangbo3504@126.com
}

How to cite this paper: Yang, B., Li, Y. and Sun, L. (2017) Analysis of Shupe Effect of Fiber Optic Ring Resonator Based on Photonic Crystal Fiber. Journal of Applied Mathematics and Physics, 5, 831-835. https://doi.org/10.4236/jamp.2017.54072

Received: March 31, 2017

Accepted: April 23, 2017

Published: April 26, 2017

\begin{abstract}
Resonator fiber optic gyroscope (RFOG) is a new kind of high precision inertial sensor based on Sagnac effect by using a shorter fiber. This paper analyzes the noise induced by Shupe effect, and the characteristics of fiber optic ring resonator (FORR) based on photonic crystal fiber are analyzed. The influence of temperature on polarization and noise induced by Shupe effect are mainly investigated, and simulation results show that FORR based on photonic crystal fiber exhibits better performance than that of conventional fiber, and simulation shows that the noise induced by Shupe effect in FORR based on photonic crystal fiber is 7 times lower than conventional fiber.
\end{abstract}

\section{Keywords}

Resonator, Photonic Crystal Fiber, Temperature, Shupe Effect

\section{Introduction}

Resonator fiber optic gyroscope (RFOG) is a novel inertial sensor which based on Sagnac effect [1] [2], and it exhibits better performance than interferometric fiber optic gyroscope (IFOG) due to its shorter fiber length, smaller dimension and longer period, et al. [3] [4]. These properties make RFOG has big potentiality in the miniaturization and integration of gyroscope system, which has been a new hotspot and investigated by many researchers recently [5] [6].

Recently, investigation shows that temperatures and tress will impair the performance of gyroscope, and even slight fluctuation can induce big noise in the output signal. This will limit the application of RFOG in high precision navigation system. Aiming the improvement of RFOG, many schemes have been proposed to optimizing the performance of gyroscope. For example, Ma et al. proposes a scheme which integrates the polarimeter and resonator together to degrade the polarization fluctuation induced by temperature and stress. And anal- 
ysis shows that the noise of this scheme is six thousand times lower than that before optimization [7]. Yu et al. analyzes the performance of air-core photonic bandgap fiber (PBG) in designing of RFOG and simulates the Kerr effect. Results show that Kerr of RFOG made by PBG exhibits better performance than that of conventional fiber, and also the velocity offset induced by Kerr effect is at least one magnitude lower [8]. Lin et al. mainly investigates the application of RFOG and they analyze the polarization fluctuation induced by cross coupler which impairs the performance of gyroscope. In their scheme $90^{\circ}$ rotation-splicing is used to overcome the polarization noise and their experimentation also prove it [9].

Nowadays, photonic crystal fiber (PCF) exhibits unique properties such as high birefringence, high nonlinearity and low confinement loss. These make PCF be a new hotspot and have been fabricated to obtain high performance in gyroscope. Investigation also show that PCF exhibits better performance in depressing of temperature fluctuation, which can be used in the optimization of RFOG [10] [11]. So in this paper, PCF is used to improve the performance of RFOG, and the Shupe effect model is obtained to investigate the temperature characteristics.

\section{Theory}

Figure 1 shows the structure of fiber optic ring resonator, of which $C, L$ and $\alpha$ denote the coupler, fiber length and transmission loss of optic fiber, respectively. According to the conservation of energy, the intensity of electric field of output can be obtained as follows [12]:

$$
\begin{aligned}
& T_{\text {coupler }}\left(\theta_{c}\right)=\sqrt{\left(1-r_{c}\right)\left(1-k_{c}\right)}\left[\begin{array}{cc}
\cos \theta_{c} & -\sin \theta_{c} \\
\sin \theta_{c} & \cos \theta_{c}
\end{array}\right] \\
& T_{\text {Loop }}(L)=\sqrt{1-\alpha L}\left[\begin{array}{cc}
\mathrm{e}^{i \beta_{x} L} & 0 \\
0 & \mathrm{e}^{i \beta_{y} L}
\end{array}\right]
\end{aligned}
$$

where $r_{c}, k_{c}$ and $\theta_{c}$ are the loss of coupler, splitting ratio and polarization angle, $\beta_{x}$ and $\beta_{y}$ are the propagation constant of both polarization axes.

After optimization, the transfer function of resonator can be obtained as follow:

$$
T_{\text {resonator }}=T_{\text {coupler }}\left(\theta_{c}\right) T_{\text {Loop }}(L)
$$

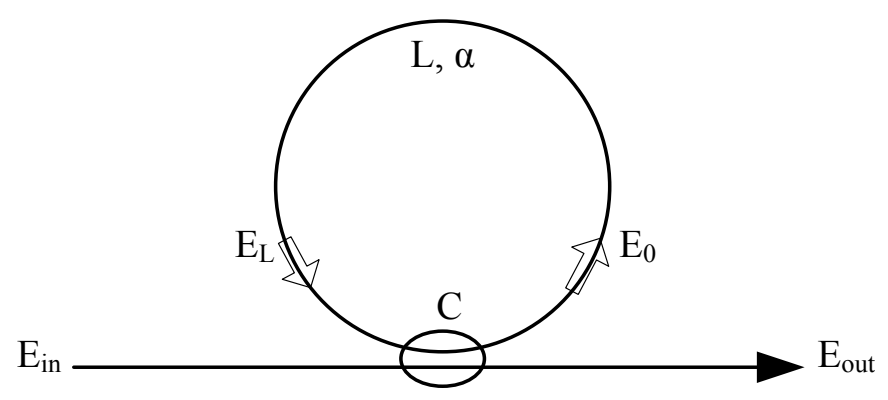

Figure 1. The structure of fiber optic ring resonator. 
From above, the output of detector can be expressed as follows:

$$
\begin{aligned}
& I_{D 1}=C\left\{\left(1-K_{c w}\right)\left[1-\rho \Gamma\left(f_{c w}\right)\right]+K_{c w}\left[1-\rho \Gamma\left(f_{c w}-\frac{\Delta \beta L}{2 \pi \tau}\right)\right]\right\} \mathrm{e}^{-\alpha_{c}} I \\
& I_{D 2}=C\left\{\left(1-K_{c c w}\right)\left[1-\rho \Gamma\left(f_{c c w}\right)\right]+K_{c c w}\left[1-\rho \Gamma\left(f_{c c w}-\frac{\Delta \beta L}{2 \pi \tau}\right)\right]\right\} \mathrm{e}^{-\alpha_{c}} I
\end{aligned}
$$

where $K_{c w}$ and $K_{c c w}$ are the power coefficient of both polarization axes, $\rho$ is the resonance depth of resonator, $\Delta \beta L$ is the phase of both state of polarization. $f_{c w}$ and $f_{c c w}$ are the frequency of light propagated in clockwise and anticlockwise direction, respectively.

When temperature fluctuated, the effective refractive index can be obtained:

$$
\Delta n^{\prime}=\frac{\partial \Delta n}{\partial T} \cdot \Delta T
$$

where $\frac{\partial \Delta n}{\partial T}$ is the temperature coefficient of PCF and $\Delta T$ is the temperature fluctuation quantity.

Then the phase due to temperature fluctuation can be expressed:

$$
\Delta \beta=\beta_{x}-\beta_{y}=\frac{2 \pi}{\lambda}\left(\Delta n+\Delta n^{\prime}\right)
$$

And the error due to the temperature can be obtained:

$$
\begin{aligned}
& \delta \Omega_{T}=\frac{n^{2} S L \Delta T}{12 a} \\
& S=\frac{1}{\phi} \frac{\mathrm{d} \phi}{\mathrm{d} t}=\left(\alpha_{1}+\frac{1}{n} \frac{\mathrm{d} n}{\mathrm{~d} T}\right)=S_{L}+S_{n}
\end{aligned}
$$

where $S$ is the Shupe constant.

\section{Results and Discussion}

In this paper, the relationship between characteristics of resonator and temperature is analyzed. And some parameters are set as: the wavelength $\lambda=1.55 \mu \mathrm{m}$, coupler coefficient $\alpha_{c}=0.05$ and the loss of fiber $\alpha=0.0023 \mathrm{~dB} / \mathrm{km}$.

\subsection{Resonance Curves}

Figure 2 shows the resonance curves of resonator of PCF and conventional fiber, from which two resonance points can be observed. It is denoted the state of polarization. After analysis, it is also found that the phase difference of both state of polarization of PCF is smaller than that of conventional fiber when under the same temperature fluctuation, which indicate that PCF has a better applicability than conventional fiber when suffer from complex condition.

\subsection{Shupe Effect}

Figure 3 shows the error curves of resonator induced by temperature, from which it is observed that the error increases with the increases of temperature 


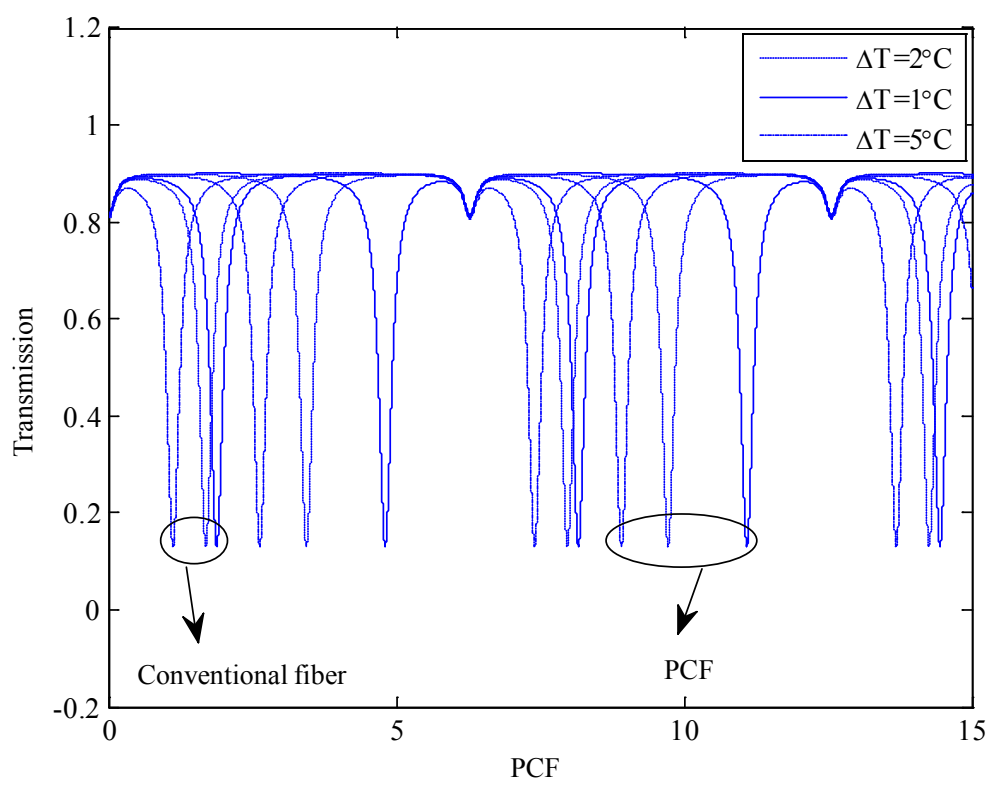

Figure 2. Influence of temperature on polarization of fiber optic ring resonator.

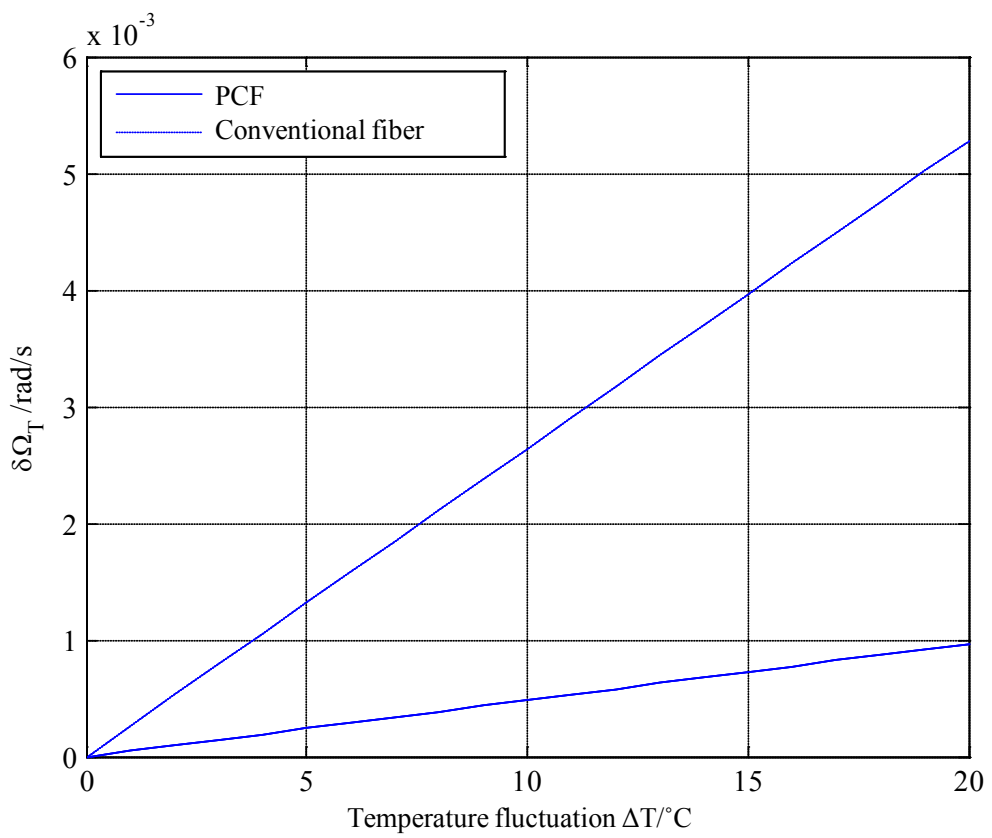

Figure 3. Influence of temperature on noise induced by Shupe effect.

fluctuation. In addition, it is also found that under the same temperature fluctuation, the error of conventional fiber is higher than that of PCF. These results show that PCF will performance well in designing and fabricating of resonator and can also improve the characteristics of gyroscope when suffer from temperature fluctuation.

\section{Conclusion}

In conclusion, PCF is used to analyze the performance of resonator and the resonance curves and the error induced by Shupe effect is obtained. Results show 
that PCF will depress the error induced by temperature fluctuation and will optimize the performance of resonator used to enhance the performance of gyroscope.

\section{References}

[1] Tang, Q.A. and Ma, X.Y. (1998) Experiment Study on Fiber Ring Resonator in Resonator Fiber Optic Gyroscope. Infrared and Laser Engineering, 27, 33-36.

[2] Zhang, X.L. and Zhou, K.J. (2009) Polarization Fluctuation in Resonator of Resonator Fiber Optic Gyro. Chinese Journal of Lasers, 36, 2083-2088. https://doi.org/10.3788/CJL20093608.2083

[3] Liu, S.R., Wu, Y.J. and $\mathrm{Xu}, \mathrm{L}$. (2008) Relationship between Vibration Error and Structural Resonance in Closed-Loop FOG. Infrared and Laser Engineering, 37, 256-259.

[4] Wang, W., Wang, X.F. and Xia, J.L. (2012) The Influence of Er-Doped Fiber Source under Irradiation on Fiber Optic Gyro. Optical Fiber Technology, 18, 39-43. https://doi.org/10.1016/j.yofte.2011.11.006

[5] Ma, H.L., He, Z.Y. and Hotate, K.Z. (2011) Reduction of Backscattering Induced Noise by Carrier Suppression in Waveguide-Type Optical Ring Resonator Gyro. Journal of Lightwave Technology, 29, 85-90. https://doi.org/10.1109/JLT.2010.2092751

[6] Hong, L.F., Zhang, C.X., Feng, L.S., et al. (2011) Research on Nonlinearity of Phase Modulation in Resonator Micro-Optic Gyro. Chinese Journal of Lasers, 38, 1105004-1-1105004-6.

[7] Ma, H.L., Yu, X.H. and Jin, Z.H. (2012) Reduction of Polarization-Fluctuation Induced Drift in Resonator Fiber Optic Gyro by a Resonator Integrating In-Line Polarizers. Optics Letters, 37, 3342-3344. https://doi.org/10.1364/OL.37.003342

[8] Yu, X.J., Liao, Y.B., Zhang, M., et al. (2008) Kerr Effect in an Optical Passive Ring Resonator Gyro Using a Hollow-Core Photonic Band-Gap Fiber. Chinese Journal of Lasers, 35, 430-435. https://doi.org/10.3788/CJL20083503.0430

[9] Lin, H.Z., Yao, Q. and Hu, Y.M. (2010) Study on 90 Docking Error Control in Polarization Maintaining Fiber Resonator of Resonant Fiber Optic Gyroscope. Acta Optica Sinica, 30, 2864-2868. https://doi.org/10.3788/AOS20103010.2864

[10] Wang, W., Yang, B. and Song, H.R., et al. (2012) Characteristics Analyses of High Birefringence and Two Zero Dispersion Points Photonic Crystal Fiber with Octagonal Lattices. Acta Physica Sinica, 61, 144601-1-144601-6.

[11] Ma, P., Song, N.F. and Jin, J. (2012) Birefringence Sensitivity to Temperature of Polarization Maintaining Photonic Crystal Fibers. Optics \& Laser Technology, 44, 1829-1833. https://doi.org/10.1016/j.optlastec.2011.12.053

[12] Wang, W. and Xia, J.L. (2011) The Characteristics of Polarization in Resonator Integrated Optic Gyroscope. Optical and Quantum Electronics, 42, 313-325. https://doi.org/10.1007/s11082-011-9464-9 
Submit or recommend next manuscript to SCIRP and we will provide best service for you:

Accepting pre-submission inquiries through Email, Facebook, LinkedIn, Twitter, etc. A wide selection of journals (inclusive of 9 subjects, more than 200 journals)

Providing 24-hour high-quality service

User-friendly online submission system

Fair and swift peer-review system

Efficient typesetting and proofreading procedure

Display of the result of downloads and visits, as well as the number of cited articles Maximum dissemination of your research work

Submit your manuscript at: http://papersubmission.scirp.org/

Or contact jamp@scirp.org 